\title{
Attitude Control Using Three Control Moment Gyros
}

\author{
By Andrew Meldrum, ${ }^{1)}$ Sho NonOmura, ${ }^{2)}$ Katsuhiko YAmadA, ${ }^{2)}$ and Yasuhiro ShoJI ${ }^{2)}$ \\ ${ }^{1)}$ School of Engineering, Osaka University, Suita, Japan \\ ${ }^{2)}$ Graduate School of Engineering, Osaka University, Suita, Japan
}

(Received June 27th, 2017)

\begin{abstract}
In the case of three-axis attitude control of spacecraft by control moment gyros (CMG), more than three CMGs are typically used for redundancy. Even when one CMG fails and the redundancy is lost, the attitude control must be maintained. In this paper, by focusing on attitude control with three CMGs, suitable steering laws are considered. In order to use the full angular momentum workspace of three CMGs, singularity problems occur. The problems occur more severely than four CMG case. Two steering laws for the four CMG pyramid configuratio with one CMG failure are proposed; Inverse Kinematics Steering Logic (IKSL) and Forward And Backward Reaching Inverse Kinematics (FABRIK). The aim of each steering law is to provide gimbal rates from the calculation of gimbal angles by inverse kinematics of the CMGs. IKSL exactly solves the inverse kinematics of the system, whereas FABRIK uses a heuristic approach to findin an approximate solution of the inverse kinematics problem. Numerical simulations are performed to validate the effectiveness of the proposed steering laws as compared with the Singular Direction Avoidance (SDA) steering law.
\end{abstract}

Key Words: Spacecraft, Attitude Control, CMG, Inverse Kinematics, FABRIK

\section{Nomenclature}

$\begin{array}{cll}\boldsymbol{A} & : & \text { Jacobian from gimbal rates to torque } \\ \boldsymbol{h}_{i} & : & \text { angular momentum of CMG- } i \\ \boldsymbol{h}_{\text {total }} & : & \text { total angular momentum of CMGs } \\ h_{\mathrm{w}} & : & \text { magnitude of wheel angular momentum } \\ \hat{\boldsymbol{g}}_{i} & : & \text { unit vector along gimbal axis of CMG- } i \\ \beta & : & \text { skew angle } \\ \boldsymbol{\theta} & : & \text { gimbal angles } \\ \boldsymbol{\tau}_{\mathrm{s}} & : & \text { attitude control torque } \\ 0 & : & \text { initial } \\ \mathrm{f} & : & \text { fina } \\ r & : & \text { reference }\end{array}$

\section{Introduction}

For communication and observation purposes, attitude control systems are needed to perform missions in space. Control moment gyros (CMGs) are momentum exchange devices to provide agile and precise attitude control of spacecraft without the use of fuel. Although they can produce a large output torque from a small input torque, they suffer singularity problems. CMGs are mainly classifie into three categories: single gimbal control moment gyros (SGCMGs), double gimbal control moment gyros (DGCMGs), and variable speed control moment gyros (VSCMGs). ${ }^{1)}$ Because of their simple mechanical structures, SGCMGs are often used for spacecraft. Because a SGCMG has only one degree of freedom, three or more SGCMGs are necessary for three-axis control. Usually an array of four or more CMGs is used to provide redundancy in the event of a failure. The most popular configuratio is a pyramidtype SGCMG system (using four SGCMGs). ${ }^{2,3)}$ Merits of this configuratio are redundancy and three-axis symmetry of output torque. While much research has been conducted on its normal operation, limited research has been presented in the case where one CMG fails and redundancy is lost. This paper will focus on these topics.

CMG arrays are subject to singularities, where a set of gimbal angles cannot realize the three-axis control torque. These singular states appear as surfaces in the momentum space of the CMG system. The redundancy of four CMGs allows for small gimbal motion to pass through the inner singular surfaces in the momentum space. However, in the case of three CMGs, larger discontinuities in the gimbal angles often occur when crossing these surfaces. These issues may be avoided by reducing the angular momentum workspace not to encounter the singular surfaces, where the inverse calculation of the Jacobian matrix from the gimbal rates to three-axis control torque cannot be calculated. However, research shows that for the commonly used skew angle of 54.7 degrees, to achieve singularity free operation, the workspace is severely limited when one CMG fails. ${ }^{4)}$ Although this restriction is relaxed by changing the skew angle, the skew angle is usually fi ed to an optimal value for the four CMG configuration Other research has investigated the fail case with magnetic torquers used in conjuction, ${ }^{5)}$ but the magnitude of the control torque is limited. To provide agile control, the entire momentum workspace should be utilized without altering the skew angle or the use of additional actuators.

The spacecraft cannot easily be repaired and refilled When one CMG fails, its wheel is spun down and attitude control with the remaining CMGs becomes necessary. The current control methods depending on the redundancy sometimes cannot be applied to the failure case. In this paper, therefore, two steering laws are proposed to provide the control when one CMG in the pyramid-type SGCMG system fails: Inverse Kinematics Steering Logic (IKSL) and Forward And Backward Reaching Inverse Kinematics (FABRIK) steering law. ${ }^{6)}$ In IKSL, the exact gimbal angles are determined from the inverse kinematics of the system to realize a desired torque. When crossing the singular surfaces, discontinuities in the gimbal angles may give rise to large gimbal rates and momentary torque errors. Even in this case, this steering law is rather accurate and its logic does not have singularities. However, IKSL is computationally ex- 
pensive and complex. On the other hand, the FABRIK steering law applies a heuristic method to solving the inverse kinematics of CMG system. Unlike IKSL which firs find all exact solutions to the inverse kinematics, FABRIK searches for only one approximate solution. It is an iterative method, where each iteration often provides an output torque closer to the desired torque. Hence, the number of iterations provides a trade-off between accuracy and computation time. While it cannot give the same tracking performance as IKSL, it is comparably simple in its implementation.

The remainder of this paper is organized as follows: In Section 2, the spacecraft model is introduced with the pyramid configuratio of four CMGs where one CMG fails and is removed. In Section 3, two new steering laws are introduced and the details of their algorithms are described. In Section 4, the results of the numerical simulations of the spacecraft attitude control with the proposed steering laws are shown and the characteristics of the simulation results are discussed. In Section 5, the conclusions of the paper are presented.

\section{Spacecraft Model}

Here, an attitude control system using three CMGs shown in Fig. 1 is focused on. This configuratio assumes one CMG fails in the popular pyramid configuratio of four CMGs.

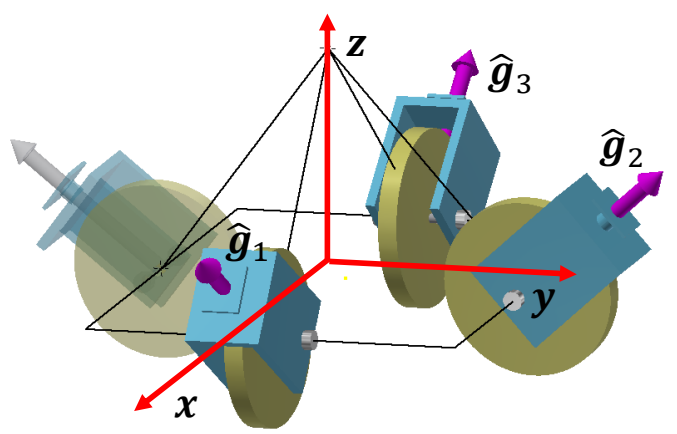

Fig. 1. Configuratio of three CMGs.

By using the skew angle $\beta$ and gimbal angle of CMG- $i$ ( $i$-th $\mathrm{CMG}), \theta_{i}$, the unit vector along the gimbal axis and that along the angular momentum of CMG- $i$ are denoted $\hat{\boldsymbol{g}}_{i}$ and $\hat{\boldsymbol{h}}_{i}$, respectively. These vectors are expressed as

$$
\begin{aligned}
{\left[\begin{array}{lll}
\hat{\boldsymbol{g}}_{1} & \hat{\boldsymbol{g}}_{2} & \hat{\boldsymbol{g}}_{3}
\end{array}\right] } & =\left[\begin{array}{ccc}
s \beta & 0 & -s \beta \\
0 & s \beta & 0 \\
c \beta & c \beta & c \beta
\end{array}\right], \\
{\left[\begin{array}{lll}
\hat{\boldsymbol{h}}_{1} & \hat{\boldsymbol{h}}_{2} & \hat{\boldsymbol{h}}_{3}
\end{array}\right] } & =\left[\begin{array}{ccc}
-s_{1} c \beta & -c_{2} & s_{3} c \beta \\
c_{1} & -s_{2} c \beta & -c_{3} \\
s_{1} s \beta & s_{2} s \beta & s_{3} s \beta
\end{array}\right],
\end{aligned}
$$

where $\sin \theta_{i}, \cos \theta_{i}, \sin \beta$, and $\cos \beta$ are abbreviated as $s_{i}, c_{i}, s \beta$, and $c \beta$, respectively. The magnitude of the angular momentum of each CMG is the same for all CMGs and is denoted by $h_{\mathrm{w}}$. The total angular momentum of three CMGs, $\boldsymbol{h}_{\text {total }}$, is given by

$$
\boldsymbol{h}_{\text {total }}=h_{\mathrm{w}}\left(\hat{\boldsymbol{h}}_{1}+\hat{\boldsymbol{h}}_{2}+\hat{\boldsymbol{h}}_{3}\right)
$$

The unit vector along the torque of CMG- $i$ is denoted by $\hat{\tau}_{i}=$ $\hat{\boldsymbol{g}}_{i} \times \hat{\boldsymbol{h}}_{i}$. The Jacobian matrix $\boldsymbol{A}$ from the gimbal rates to the control torque is given by

$$
\boldsymbol{A}=\left[\begin{array}{lll}
\hat{\boldsymbol{\tau}}_{1} & \hat{\boldsymbol{\tau}}_{2} & \hat{\boldsymbol{\tau}}_{3}
\end{array}\right]=\left[\begin{array}{ccc}
-c_{1} c \beta & s_{2} & c_{3} c \beta \\
-s_{1} & -c_{2} c \beta & s_{3} \\
c_{1} s \beta & c_{2} s \beta & c_{3} s \beta
\end{array}\right] .
$$

By using the Jacobian $\boldsymbol{A}$, the attitude control torque $\tau_{\mathrm{s}}$ generated by the CMGs is given by

$$
\boldsymbol{\tau}_{\mathrm{s}}=-h_{\mathrm{w}} \boldsymbol{A} \dot{\boldsymbol{\theta}}, \quad \boldsymbol{\theta}=\left[\begin{array}{c}
\theta_{1} \\
\theta_{2} \\
\theta_{3}
\end{array}\right] .
$$

The gimbal rates of the CMGs, $\dot{\boldsymbol{\theta}}$, can be calculated directly from Eq. (5),

$$
\dot{\boldsymbol{\theta}}=-\frac{1}{h_{\mathrm{w}}} \boldsymbol{A}^{-1} \boldsymbol{\tau}_{\mathrm{s}}
$$

When $\operatorname{det} \boldsymbol{A}=0, \boldsymbol{A}^{-1}$ cannot be calculated and the CMGs are in the singular state. In this paper, in order to avoid the singularity issues, $\dot{\boldsymbol{\theta}}$ is obtained by using inverse kinematics that calculates the gimbal angles from the total angular momentum of the CMGs.

\section{Steering Laws}

Although most steering laws for attitude control the CMGs have singularity issues, inverse kinematics methods have no singularities because these methods calculate $\dot{\theta}$ from the gimbal angles $\boldsymbol{\theta}$. In the following section, two steering laws are introduced. One is a comparatively analytical and accurate method by a complex calculation. Another is a heuristic method by a simple calculation.

\subsection{Inverse kinematics steering $\operatorname{logic^{2}}$}

Inverse kinematics steering logic (IKSL) uses the inverse kinematics of CMGs. Generally, the inverse kinematics provides the inputs from the given outputs. In the CMG case, outputs are total angular momentum of CMGs whereas inputs are the gimbal angles of the CMGs. The solutions are numerically calculated because the analytical solution cannot be obtained.

\subsubsection{Algorithm}

As described above, the inverse kinematics of CMGs is to fin $\boldsymbol{\theta}$ from the given $\boldsymbol{h}_{\text {total }}$. The elements of the given $\boldsymbol{h}_{\text {total }}$ is expressed by $\boldsymbol{h}_{\text {total }}=h_{\mathrm{w}}\left[\begin{array}{lll}h_{\mathrm{x}} & h_{\mathrm{y}} & h_{\mathrm{z}}\end{array}\right]^{T}$. The problem is to fin the solution of the following equations:

$$
\begin{aligned}
-s_{1} c \beta-c_{2}+s_{3} c \beta & =h_{\mathrm{x}}, \\
c_{1}-s_{2} c \beta-c_{3} & =h_{\mathrm{y}}, \\
s_{1} s \beta+s_{2} s \beta+s_{3} s \beta & =h_{\mathrm{z}} .
\end{aligned}
$$

From Eqs. (7)-(9), the following equation for $s_{2}$ and $c_{2}$ is obtained by eliminating $s_{1}, c_{1}, s_{3}$, and $c_{3}$ as:

$$
\begin{aligned}
& {\left[\left(h_{\mathrm{y}}+s_{2} c \beta\right)^{2}+\left(\frac{h_{\mathrm{x}}+c_{2}}{c \beta}\right)^{2}\right]\left[\left(h_{\mathrm{y}}+s_{2} c \beta\right)^{2}+\left(\frac{h_{\mathrm{z}}-s_{2} s \beta}{s \beta}\right)^{2}\right]} \\
& =4\left(h_{\mathrm{y}}+s_{2} c \beta\right)^{2}
\end{aligned}
$$

This equation is reduced to an eighth-order polynomial equation for $s_{2}$. Because the equation cannot be solved analytically, the solution for $s_{2}$ is obtained numerically. Once the solution for 
$s_{2}$ is obtained, the solutions for $c_{2}, s_{1}, c_{1}, s_{3}$, and $c_{3}$ are easily calculated from the following equations:

$$
\begin{aligned}
& s_{1}^{2}+c_{1}^{2}=1, \\
& s_{2}^{2}+c_{2}^{2}=1, \\
& s_{3}^{2}+c_{3}^{2}=1, \\
& s_{1}=\frac{-\left(s_{2} c \beta+c_{2}+h_{\mathrm{x}}\right) s \beta+h_{\mathrm{z}} c \beta}{2 s \beta c \beta}, \\
& s_{3}=\frac{\left(-s_{2} c \beta+c_{2}+h_{\mathrm{x}}\right) s \beta+h_{\mathrm{z}} c \beta}{2 s \beta c \beta}, \\
& c_{1}-c_{3}=h_{\mathrm{y}}+s_{2} c \beta .
\end{aligned}
$$

The gimbal angle $\theta_{i}$ is uniquely calculated from each set of $s_{i}$ and $c_{i}$, and $\boldsymbol{\theta}$ has at most eight solutions.

\subsubsection{Gimbal rate limitation}

In the feedback attitude control, a reference quaternion and a reference angular velocity of the spacecraft are generated first The attitude control torque $\tau_{\mathrm{s}}$ is calculated from the difference between the current and the reference states. The time interval and the variation of the total angular momentum of CMGs at each interval are denoted by $\Delta t$ and $\Delta \boldsymbol{h}_{\text {total }}$, respectively. The target value of the total angular momentum $\tilde{\boldsymbol{h}}_{\text {total }}$ is given by

$$
\tilde{\boldsymbol{h}}_{\text {total }}=\boldsymbol{h}_{\text {total }}+\Delta \boldsymbol{h}_{\text {total }}=\boldsymbol{h}_{\text {total }}-\boldsymbol{\tau}_{\mathrm{s}} \Delta t
$$

Because the inverse kinematics has eight or fewer solutions, the selection among the obtained solutions is needed. The present gimbal angles and the solution of the inverse kinematics for $\tilde{\boldsymbol{h}}_{\text {total }}$ are denoted by $\boldsymbol{\theta}$ and $\tilde{\boldsymbol{\theta}}$, respectively. Here, $\|\tilde{\boldsymbol{\theta}}-\boldsymbol{\theta}\|$ is minimized to select the appropriate solution. The variation of the gimbal angle is calculated by $\Delta \boldsymbol{\theta}=\tilde{\boldsymbol{\theta}}-\boldsymbol{\theta}$. If the CMG state is in the inner impassable singular surfaces, the ideal $\tilde{\boldsymbol{\theta}}$ cannot be realized because $\Delta \boldsymbol{\theta}$ becomes too large for one sampling interval. In such a case, by using the limit of $\|\Delta \boldsymbol{\theta}\|, \Delta \theta_{\max }, \dot{\boldsymbol{\theta}}$ is determined as follows:

$$
\dot{\boldsymbol{\theta}}=\frac{1}{\Delta t} \min \left(\Delta \theta_{\max },\|\Delta \boldsymbol{\theta}\|\right) \frac{\Delta \boldsymbol{\theta}}{\|\Delta \boldsymbol{\theta}\|}
$$

When $\dot{\boldsymbol{\theta}}$ is given by Eq. (18), $\boldsymbol{\theta}$ does not reach $\tilde{\boldsymbol{\theta}}$ in one sampling interval. Even in this case, IKSL helps the CMGs to pass through the inner impassable singular state.

\subsection{FABRIK steering logic}

In this subsection, a heuristic iterative method, Forward And Backward Reaching Inverse Kinematics (FABRIK), ${ }^{6)}$ is introduced and modifie for the inverse kinematics of CMGs. In the case of IKSL, because the solutions are obtained directly from a complicated equation (8th order polynomial equation), the solutions are accurate but the procedure is complicated. Especially when the CMG configuratio has additional degrees of freedom, it is rather difficult to select the best solution. Using FABRIK, the solutions are not always accurate but simple. FABRIK can calculate the solution easily in the same way even if the configuratio has four or more CMGs.

\subsubsection{Algorithm}

A temporary angular momentum $\boldsymbol{p}_{i}$ is define by the summation of the angular momentum of each CMG as follows:

$$
\begin{aligned}
\boldsymbol{p}_{1} & =\mathbf{0}, \\
\boldsymbol{p}_{2} & =h_{\mathrm{w}} \hat{\boldsymbol{h}}_{1}, \\
\boldsymbol{p}_{3} & =h_{\mathrm{w}}\left(\hat{\boldsymbol{h}}_{1}+\hat{\boldsymbol{h}}_{2}\right), \\
\boldsymbol{p}_{4} & =h_{\mathrm{w}}\left(\hat{\boldsymbol{h}}_{1}+\hat{\boldsymbol{h}}_{2}+\hat{\boldsymbol{h}}_{3}\right) .
\end{aligned}
$$

The initial values of $\boldsymbol{p}_{0}$ and $\boldsymbol{p}_{4}$ are set at $\mathbf{0}$ and $\tilde{\boldsymbol{h}}_{\text {total }}$, respectively; $\boldsymbol{p}_{1}=\mathbf{0}, \boldsymbol{p}_{4}=\tilde{\boldsymbol{h}}_{\text {total }}$. In FABRIK, these two equations are alternately substituted in the backward and forward procedures, and each procedure is calculated as follows: The distance between each $\boldsymbol{p}_{i}$ is firstl define by $\boldsymbol{r}_{i}=\boldsymbol{p}_{i+1}-\boldsymbol{p}_{i}$. First, let $\boldsymbol{p}_{4}$ be $\tilde{\boldsymbol{h}}_{\text {total }}$ and the backward calculation is executed. The vector $\boldsymbol{r}_{i}$ is modifie so that $\boldsymbol{p}_{i}$ is realized by the angular momentum $\boldsymbol{h}_{i}$. The new distance $\boldsymbol{r}_{i}$ is calculated by projecting $\boldsymbol{r}_{i}$ onto an orthogonal plane to $\boldsymbol{g}_{i}$ and changing the size to the magnitude of angular momentum of each CMG, $h_{\mathrm{w}}$. That is, $\boldsymbol{r}_{i}$ is modifie as follows:

$$
\boldsymbol{r}_{i}=h_{\mathrm{w}} \hat{\boldsymbol{r}}_{i}, \quad \hat{\boldsymbol{r}}_{i}=\frac{\boldsymbol{r}_{i}-\left(\boldsymbol{r}_{i} \cdot \boldsymbol{g}_{i}\right) \boldsymbol{g}_{i}}{\left\|\boldsymbol{r}_{i}-\left(\boldsymbol{r}_{i} \cdot \boldsymbol{g}_{i}\right) \boldsymbol{g}_{i}\right\|}
$$

The gimbal angle of CMG $-i$ is calculated from $\boldsymbol{r}_{i}$. The difference angle between the calculated gimbal angle of CMG- $i$ and the current one, $\theta_{i \text { dif }}$, is obtained from

$$
\sin \theta_{i \mathrm{dif}}=\left(\hat{\boldsymbol{h}}_{i} \times \hat{\boldsymbol{r}}_{i}\right) \cdot \hat{\boldsymbol{g}}_{i}, \quad \cos \theta_{i \mathrm{dif}}=\hat{\boldsymbol{h}}_{i} \cdot \hat{\boldsymbol{r}}_{i} .
$$

If $\theta_{i \text { dif }}$ is larger than the limited value, $\boldsymbol{r}_{i}$ is recalculated as follows:

$$
\boldsymbol{r}_{i}= \begin{cases}\boldsymbol{r}_{i} & \left|\theta_{i \mathrm{dif}}\right| \leq \theta_{\max } \\ h_{\mathrm{w}}\left(c_{\mathrm{m}} \hat{\boldsymbol{h}}_{i}+\operatorname{sign}\left(\theta_{i \mathrm{dif}}\right) s_{\mathrm{m}} \hat{\boldsymbol{g}}_{i} \times \hat{\boldsymbol{h}}_{i}\right) & \left|\theta_{i \mathrm{dif}}\right|>\theta_{\max }\end{cases}
$$

where $\theta_{\max }$ is the allowable magnitude of $\theta_{i \text { dif }}$ and $\sin \theta_{\max }$ and $\cos \theta_{\max }$ are abbreviated as $s_{\mathrm{m}}$ and $c_{\mathrm{m}}$, respectively. Then, $\boldsymbol{p}_{i}$ is redefine as $\boldsymbol{p}_{i+1}-\boldsymbol{r}_{i}$. This calculation is executed from $i=3$ to $i=2$. Second, let $\boldsymbol{p}_{1}$ be $\mathbf{0}$ and the forward calculation is executed from $i=1$ to $i=2$. As shown in the backward procedure, $\boldsymbol{r}_{i}$ is calculated by projecting $\boldsymbol{p}_{i+1}-\boldsymbol{p}_{i}$ onto an orthogonal plane to $\hat{\boldsymbol{g}}_{i}$ and changing the size to the magnitude of angular momentum of each CMG, $h_{\mathrm{w}}$. If the difference angle $\theta_{i \mathrm{dif}}$ is larger than the limited value, Eq. (25) is also applied in this procedure. Then, $\boldsymbol{p}_{i+1}$ is redefine as $\boldsymbol{p}_{i}+\boldsymbol{r}_{i}$. Therefore, $\boldsymbol{p}_{i}$ is calculated in the backward and forward procedure as follows:

$$
\begin{array}{r}
\text { Backward procedure : } \boldsymbol{p}_{i}=\boldsymbol{p}_{i+1}-\boldsymbol{r}_{i} \\
\text { Forward procedure : } \boldsymbol{p}_{i+1}=\boldsymbol{p}_{i}+\boldsymbol{r}_{i}
\end{array}
$$

By repeating the backward and forward procedures a certain number of times, $\boldsymbol{p}_{4}$ is finall calculated and it becomes positioned near the target. The angular momentum of each $\mathrm{CMG}-i$ is calculated from $\boldsymbol{p}_{i+1}$ and $\boldsymbol{p}_{i}$, and the whole procedure of FABRIK is shown in Fig. 2. 


\subsubsection{Modification of initial $\theta$}

In this subsection, initial setting of $\boldsymbol{\theta}$ is considered. FABRIK can get only one solution near the current gimbal angles because it searches the solution within the limit value from the current gimbal angles. In the singular state, it sometimes takes a lot of time to pass through the singular surface. In FABRIK, the selection of the initial gimbal angles is important to obtain the proper gimbal angle solution in an allowable number of iterations.

Here, the initial angular momentum is calculated from the modifie gimbal angles $k_{\text {ini }} \boldsymbol{\theta}$. The parameter $k_{\text {ini }}$ is a constant value for changing the initial gimbal angles from $\boldsymbol{\theta}$, and in the case of $k_{\text {ini }}<1$, the solution search is started from the smaller gimbal angles than the current ones. In the case of three CMGs, there are only a limited number of solutions since the configu ration has no redundancy. Although the terminal gimbal state in the attitude maneuver is different from the initial one unless specificall devised, the terminal state sometimes becomes the same as the initial state in FABRIK with $k_{\text {ini }}<1$. This is because the solution of $\boldsymbol{\theta}$ is likely to be small in the case of $k_{\text {ini }}<1$. Returning to the initial condition is desirable in the case of the repetition of attitude maneuvers, and in some cases (but not always), setting $k_{\text {ini }}<1$ helps the terminal gimbal angles become the same as the initial ones.

\subsection{Modification to three CMG configuration}

In this subsection, modification of the algorithms of IKSL and FABRIK from those of the four CMG pyramid configu ration to those of three $\mathrm{CMG}$ configuratio are summarized. When one CMG fails in the four CMG configuration the remaining degrees of freedom is three and the control redundancy is lost. In the case of IKSL, there are infinit solutions for the gimbal angles in the inverse kinematics of the four CMG configuration whereas there are at most eight solutions of the three CMG configuration In the case of the four CMG configura tion, there must be an optimization procedure to select the best solution among the infinit solutions. Therefore, the IKSL algorithm is simpler in the three CMG configuratio than that in the four CMG configuration In the case of FABRIK, the computation procedure is almost the same in the four and three CMG configurations This is one of the merits of FABRIK, and the computation time is almost proportional to the number of CMGs.

\section{Numerical Simulations}

In this section, maneuver simulations are executed in order to examine the capabilities of IKSL and FABRIK. For the comparison, a simple steering law named Singular Direction Avoidance $(\mathrm{SDA})^{7)}$ is also applied.

\subsection{Attitude control}

The spacecraft attitude is expressed by Euler parameters. Euler parameters $\boldsymbol{q}$ are define by the rotation angle $\theta_{\mathrm{s}}$ around the Euler axis $\hat{\boldsymbol{a}}$ as follows:

$$
\boldsymbol{q}\left(\theta_{\mathrm{s}}, \boldsymbol{a}\right)=\left[\begin{array}{c}
S(\boldsymbol{q}) \\
V(\boldsymbol{q})
\end{array}\right]=\left[\begin{array}{c}
\cos \frac{\theta_{\mathrm{s}}}{2} \\
\hat{\boldsymbol{a}} \sin \frac{\theta_{\mathrm{s}}}{2}
\end{array}\right],
$$

where $S(\boldsymbol{q})$ and $V(\boldsymbol{q})$ refer to the scalar part and the vector part, respectively. In the simulations, the spacecraft is controlled by

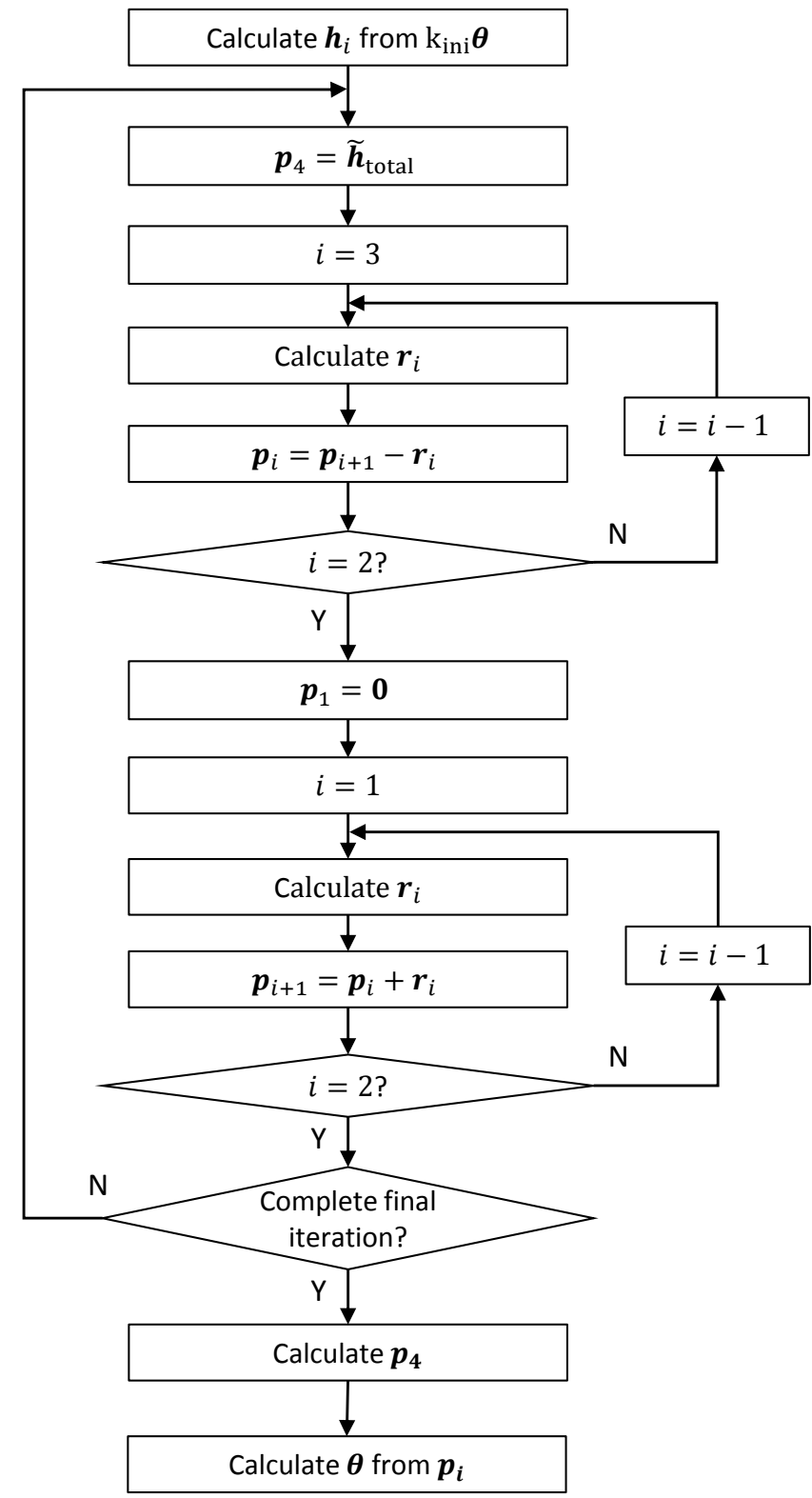

Fig. 2. Flowchart of FABRIK.

a PD control law so that its Euler parameters and angular velocity follow the reference Euler parameters $\boldsymbol{q}_{\mathrm{r}}$, and the reference angular velocity $\omega_{\mathrm{r}}$, respectively. Let $\boldsymbol{q}_{\mathrm{r}}$ and $\omega_{\mathrm{r}}$ be expressed by the half rotation time, $t_{\mathrm{f}}, \hat{\boldsymbol{a}}$, and $\theta_{\mathrm{s}}$ as follows:

$$
\begin{aligned}
& \omega_{\mathrm{r}}(t)= \begin{cases}\frac{\theta_{\mathrm{sf}}}{t_{\mathrm{f}}^{2}} \hat{\boldsymbol{a}} t & t<t_{\mathrm{f}} \\
\frac{2 \theta_{\mathrm{sf}}}{t_{\mathrm{f}}} \hat{\boldsymbol{a}}-\frac{\theta_{\mathrm{sf}}}{t_{\mathrm{f}}^{2}} \hat{\boldsymbol{a}} t & t_{\mathrm{f}}<t<2 t_{\mathrm{f}} \\
\mathbf{0} & 2 t_{\mathrm{f}}<t\end{cases} \\
& \dot{\boldsymbol{q}}_{\mathrm{r}}(t)=\frac{1}{2} \boldsymbol{q}_{\mathrm{r}}(t) \otimes \omega_{\mathrm{r}}(t),
\end{aligned}
$$

where $\otimes$ denotes a quaternion multiplication. An example of time histories of $\boldsymbol{q}_{\mathrm{r}}$ and $\omega_{\mathrm{r}}$ is shown in Fig. 3 where $t_{\mathrm{f}}, \hat{\boldsymbol{a}}$, and $\theta_{\mathrm{s}}$ are set at the values in Table 1 .

The proportional gain and the derivative gain are described by $k_{\mathrm{p}}$ and $k_{\mathrm{d}}$, respectively. The attitude control torque $\tau_{\mathrm{s}}$ is define by the error of the Euler parameters $\boldsymbol{q}_{\mathrm{e}}$ and the error of the 


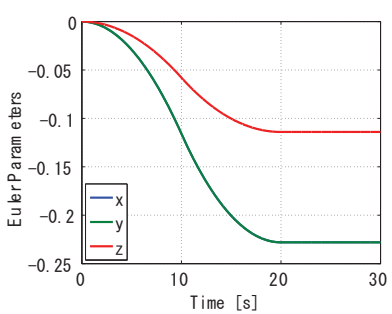

Euler parameter $\boldsymbol{q}_{\mathrm{r}}$.

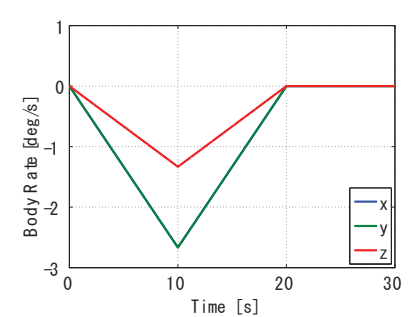

Angular velocity $\omega_{\mathrm{r}}$
Fig. 3. Reference trajectories

angular velocity $\omega_{\mathrm{e}}$ as follows:

$$
\tau_{\mathrm{s}}=-k_{\mathrm{p}} V\left(\boldsymbol{q}_{\mathrm{e}}\right)-k_{\mathrm{d}} \omega_{\mathrm{e}}
$$

where

$$
\begin{aligned}
& \boldsymbol{q}_{\mathrm{e}}=\boldsymbol{q}_{\mathrm{r}}^{\dagger} \otimes \boldsymbol{q}, \\
& \omega_{\mathrm{e}}=\omega-\omega_{\mathrm{r}},
\end{aligned}
$$

and $\boldsymbol{q}_{\mathrm{r}}^{\dagger}$ denotes the conjugate quaternion of $\boldsymbol{q}_{\mathrm{r}}$. In the proposed steering laws, IKSL and FABRIK, the angular momentum command $\tilde{\boldsymbol{h}}_{\text {total }}$ is given by Eq. (17).

In order to compare the performances of the steering laws in the singular state, a tracking maneuver is designed so that the total angular momentum passes through an impassable singular surface. The moment of inertia of the spacecraft, $\boldsymbol{J}$, is set as follows:

$$
\boldsymbol{J}=\left[\begin{array}{ccc}
0.5541 & 0.0130 & 0.0183 \\
0.0130 & 0.5447 & -0.0149 \\
0.0183 & -0.0149 & 0.6289
\end{array}\right]\left[\mathrm{kgm}^{2}\right] .
$$

\begin{tabular}{|c|c|}
\hline Items & Values \\
\hline Moment of inertia & $J$ \\
\hline Wheel angular momentum & $h_{\mathrm{w}}=0.0576[\mathrm{Nms}]$ \\
\hline Skew angle of each CMG & $\beta=45[\mathrm{deg}]$ \\
\hline Initial gimbal angles & $\boldsymbol{\theta}=[-45,0,45]^{T}[\mathrm{deg}]$ \\
\hline Eular axis & $\hat{\boldsymbol{a}}=[-2 / 3,-2 / 3,-1 / 3]^{T}$ \\
\hline Proportional gain & $k_{\mathrm{p}}=10[\mathrm{Nm}]$ \\
\hline Derivative gain & $k_{\mathrm{d}}=5[\mathrm{Nms} / \mathrm{rad}]$ \\
\hline Initial Euler angle & $\theta_{\mathrm{s} 0}=0[\mathrm{deg}]$ \\
\hline Final Euler angle & $\theta_{\mathrm{sf}}=40[\mathrm{deg}]$ \\
\hline Half rotation time & $t_{\mathrm{f}}=10[\mathrm{~s}]$ \\
\hline Maximum gimbal rate & $\dot{\theta}_{\max }=2[\mathrm{rad} / \mathrm{s}]$ \\
\hline Time interval & $\Delta t=0.01[\mathrm{~s}]$ \\
\hline Parameter in SDA & $\alpha_{0}=0.1$ \\
\hline Parameter in SDA & $k_{\sigma}=10$ \\
\hline Parameter in IKSL & $\Delta \theta_{\max }=0.02[\mathrm{rad}]$ \\
\hline Parameter in FABRIK & $k_{\text {ini }}=0.8$ \\
\hline Parameter in FABRIK & $\theta_{\max }=0.02[\mathrm{rad}]$ \\
\hline
\end{tabular}

The parameters of the simulations are specifie in Table 1 .

\subsection{SDA steering logic}

The SDA steering logic is also tested in the simulations. Let the singular value decomposition of matrix $\boldsymbol{A}$ be expressed by

$$
\boldsymbol{A}=\boldsymbol{U} \boldsymbol{S} \boldsymbol{V}^{T} .
$$

In SDA, by using matrices $\boldsymbol{U}, \boldsymbol{S}$, and $\boldsymbol{V}$, the inverse matrix of matrix $\boldsymbol{A}$ is calculated as follows:

$$
\boldsymbol{A}_{\mathrm{SDA}}^{-1}=\boldsymbol{V} \boldsymbol{S}_{\mathrm{SDA}}^{-1} \boldsymbol{U}^{T}
$$

In the above equation, $\boldsymbol{S}_{\mathrm{SDA}}^{-1}$ is calculated as follows:

$$
\boldsymbol{S}_{\mathrm{SDA}}^{-1}=\operatorname{diag}\left(\frac{1}{s_{11}}, \frac{1}{s_{22}}, \frac{s_{33}}{s_{33}^{2}+\alpha}\right),
$$

where $s_{i i}$ means the singular value of matrix $\boldsymbol{A}$, that is, the $(i, i)$ th component of matrix $\boldsymbol{S}$, and the inequality $s_{11} \geq s_{22} \geq s_{33}$ is assumed. The positive parameter $\alpha$ avoids the sigularity of inverse matrix $\boldsymbol{A}^{-1}$ in the case of singular states, and is given by

$$
\alpha=\alpha_{0} e^{-k_{\sigma} s_{33}^{2}},
$$

where $\alpha_{0}$ and $k_{\sigma}$ are positive constants.

The torque errors are produced by the parameter $\alpha$ in the singular direction expressed by the third column of matrix $\boldsymbol{U}$ and its effects become larger near the singular surface. On the singular surface, the gimbal rates become zeros in the singular direction, and thus, passing through the singular surface sometimes becomes difficult in SDA.

\subsection{Simulation results}

Figures 4 - 6 show the simulation results of SDA, IKSL, and FABRIK, respectively. In each figure the firs subfigur shows vector part of the Euler parameters $V(\boldsymbol{q})$. The second shows vector part of the error Euler parameters $V\left(\boldsymbol{q}_{\mathrm{e}}\right)$. The third shows angular velocity $\boldsymbol{\omega}$. The fourth shows gimbal angles $\boldsymbol{\theta}$. The fift shows gimbal angle rates $\dot{\boldsymbol{\theta}}$. The sixth shows the condition number of $\boldsymbol{A}$.

In the case of SDA in Fig. 4, the condition number shows that the CMGs encounter a singular state at $t=10[\mathrm{~s}]$ and cannot escape until $t=25[\mathrm{~s}]$. In the singular state, the gimbal angle motion is suppressed and it takes a longer time to escape from the singular state. While the CMGs are stuck in the singular state, small torque errors resulting from parameter $\alpha$ in the singular direction accumulate, which makes the attitude error and the angular velocity error larger than those of other cases.

In the case of IKSL in Fig. 5, the gimbal angles move rapidly at $t=9[\mathrm{~s}]$ and $t=11[\mathrm{~s}]$. From the condition number, the CMGs encounter the singular state at these times but little time is spent in the singular state. Although momentary errors are observed in the angular velocity at these timings of the singular state, IKSL is best able to follow the reference trajectory with the smallest peak errors.

In the case of FABRIK in Fig. 6, the condition number shows that the CMGs are not close to the singular state. The gimbal angle motion is visibly smoother and the gimbal angle rates are also small. Although the attitude error in FABRIK is as large as that of SDA, the gimbal angle rates are much smaller than those of SDA.

All steering laws produce identical motion until a singular surface is encountered. For this trajectory, IKSL and FABRIK can return the gimbal angles to their initial state. Many other trajectories have been tested by FABRIK with $k_{\text {ini }}<1$, and in many cases, the fina gimbal angles return to the initial state though they do not always return to the initial one. These characteristics are regarded as one of the merits of FABRIK.

The singular states of CMGs are classifie into passable and impassable states, where the CMGs cannot escape from the impassable singular states directly by continuous gimbal angle motion. In the three CMG configuration the singular states become impassable because of lack of redundancy. In the simulation case, the ideal gimbal motion which realizes the angular 
Trans. JSASS Aerospace Tech. Japan Vol. 16, No. 5 (2018)

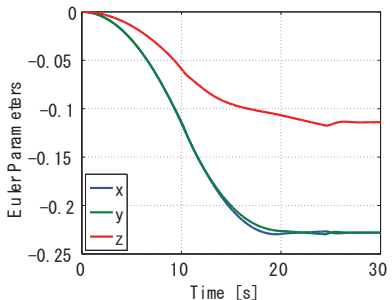

Vector part of $\boldsymbol{q}$.

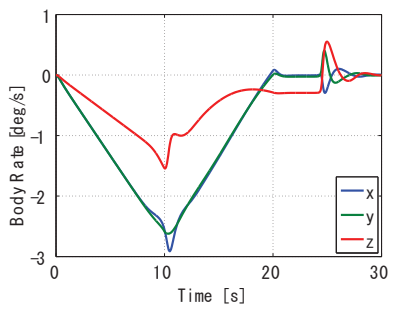

Angular velocity $\omega$

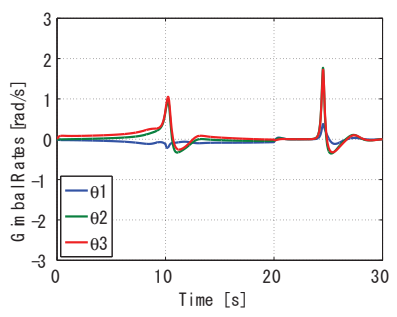

Gimbal angles rates $\dot{\boldsymbol{\theta}}$.

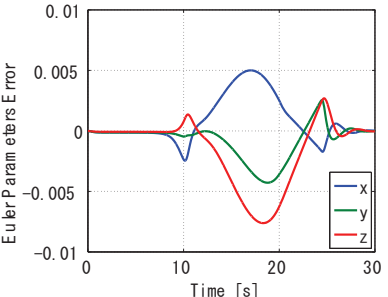

Vector part of $\boldsymbol{q}_{\mathrm{e}}$.

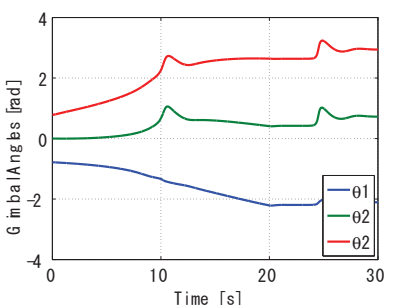

Gimbal angles $\boldsymbol{\theta}$

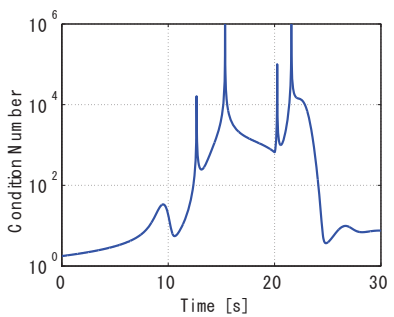

Condition number of $\boldsymbol{A}$.

Fig. 4. Attitude control simulation (SDA).

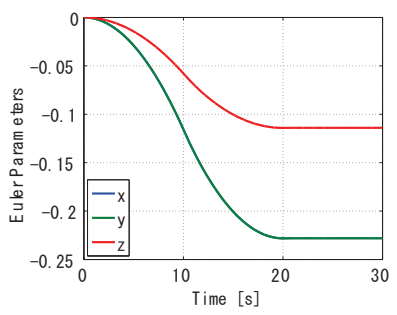

Vector part of $\boldsymbol{q}$

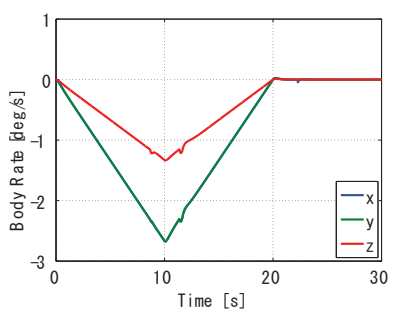

Angular velocity $\omega$.

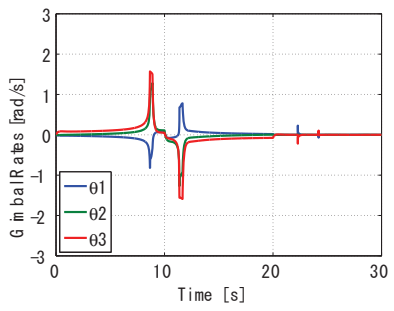

Gimbal angles rates $\dot{\boldsymbol{\theta}}$.

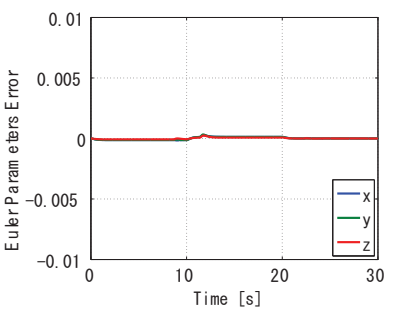

Vector part of $\boldsymbol{q}_{\mathrm{e}}$.

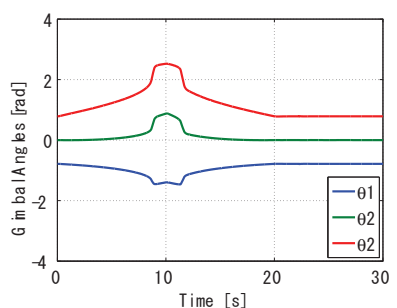

Gimbal angles $\boldsymbol{\theta}$

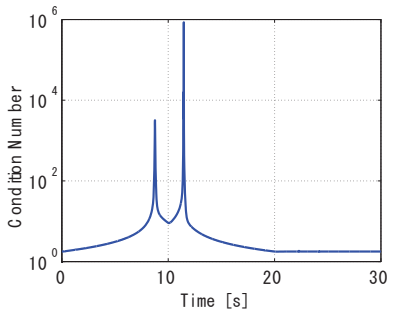

Condition number of $\boldsymbol{A}$.

Fig. 5. Attitude control simulation (IKSL).

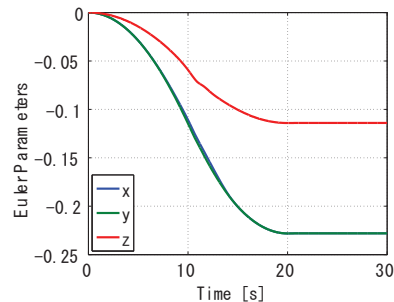

Vector part of $\boldsymbol{q}$.

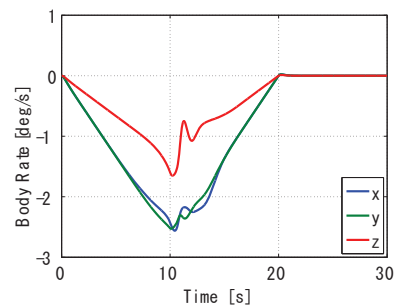

Angular velocity $\omega$.

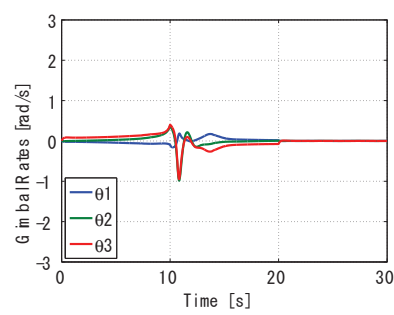

Gimbal angles rates $\dot{\boldsymbol{\theta}}$.

Fig. 6. Attitude control simulation (FABRIK).

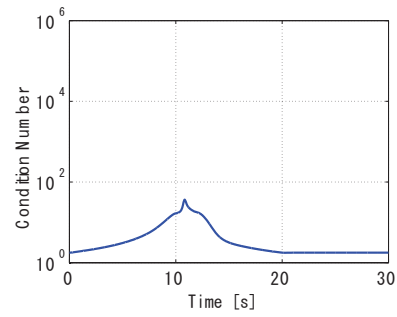

Condition number of $\boldsymbol{A}$.

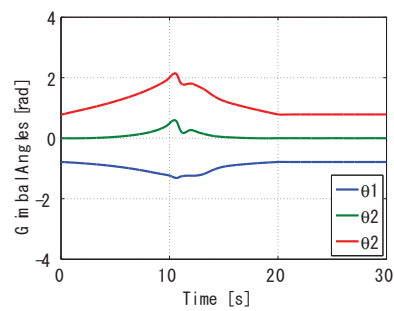

Gimbal angles $\boldsymbol{\theta}$

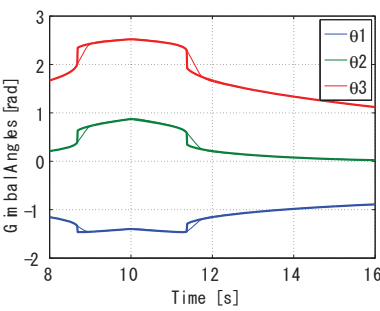

IKSL.

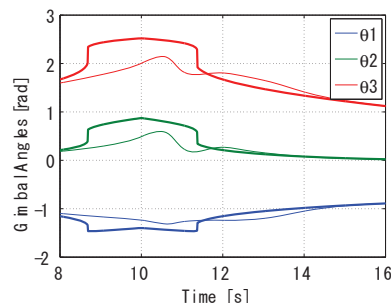

FABRIK.

Fig. 7. Gimbal angles $\boldsymbol{\theta}$ near singular states.

Table 2. Time for steering laws.

\begin{tabular}{lcc}
\hline Steering law & Average time $[\mu \mathrm{s}]$ & Maximum time $[\mu \mathrm{s}]$ \\
\hline SDA & 13.82 & 52.28 \\
IKSL & 221.97 & 523.12 \\
FABRIK & 54.56 & 138.81 \\
\hline
\end{tabular}

velocity in Eq. (29) encounters two impassable singular states. Figure 7 shows the gimbal angle behaviors near the singular states. Figure 7 shows the gimbal angle behavior of IKSL and that of FABRIK. In each figure the thick lines show the ideal gimbal motion and the thin lines show the simulated one which is identical with the gimbal angle motions in Figs. 5 and 6. The ideal gimbal angle motion jumps at $t=8.68$ [s] and $t=11.37$ [s] when the CMGs encounter the impassable singular states. In the case of IKSL, the simulated gimbal angles almost follow the ideal gimbal angles except for the vicinity of the impassable singular states. In the vicinity of the singular states, the simulated gimbal angles deviate from the ideal ones because the gimbal rates are restricted within the gimbal rate limit as shown in Eq. (18). However, this deviation is small and the CMGs can 
escape from the impassable singular states smoothly. On the other hand, in the case of FABRIK, the simulated gimbal angles deviate more largely than the case of IKSL, and therefore, the attitude errors in the vicinity of the singular states become larger. In this case, the CMGs avoid the impassable singular states by the large deviation of the gimbal angles.

To summarize the performances of the steering laws, IKSL produced the best results. However, IKSL needs much more computations than the other methods. Average and maximum computation time among these steering laws are shown in Table 2 when they are run on MATLAB with an i7-6700 CPU. In this table, the number of the iterations in FABRIK is 10 which is also used in the simulations. As shown in Table 2, the computation time of IKSL is almost four times larger than that of FABRIK, and is ten times more larger than that of SDA.

\section{Conclusions}

This paper investigates the attitude control of spacecraft using three CMGs where one CMG fails in the pyramid configu ration of four CMGs. Two steering laws based on inverse kinematics which calculates the gimbal angles of the CMGs from the total angular momentum are proposed; Inverse Kinematics Steering Logic (IKSL) and Forward And Backward Reaching Inverse Kinematics (FABRIK). IKSL is a method to obtain exact gimbal angle solutions from an eighth-order polynomial equation. The method realizes accurate attitude motion, but the computational burden is larger than FABRIK. On the other hand, FABRIK is a heuristic method to obtain the approx- imate solution by an iterative procedure. Some modification are added to the original FABRIK to be suitable for the inverse kinematics of the CMGs. Although the accuracy of the attitude motion is inferior to that of IKSL, FABRIK is easy in implementation and the computational burden is also less than IKSL. These steering laws are examined by numerical simulation in comparison with the Singular Direction Avoidance steering law (SDA). The simulation results show the validity of the proposed steering laws in escaping from the singular state.

\section{References}

1) Jikuya, I., Fujii, K., and Yamada, K.: Attitude Maneuver of Spacecraft with a Variable-Speed Double-Gimbal Control Moment Gyro, Advances in Space Research, 58 (2016), pp. 1303-1317.

2) Yamada, K., Asai, T., and Jikuya, I.: Inverse Kinematics in PyramidType Single-Gimbal Control Moment Gyro System, J. Guidance, Control, and Dynamics, 39 (2016), pp. 1897-1907.

3) Yamada, K. and Jikuya, I.: Directional Passability and Quadratic Steering Logic for Pyramid-Type Single Gimbal Control Moment Gyros, Acta Astronautica, 102 (2014), pp. 103-123.

4) Sands, T., Kim, J. J., and Agrawal, B. N.: Nonredundant SingleGimbaled Control Moment Gyroscopes, J. Guidance, Control, and Dynamics, 35 (2016), pp. 578-587.

5) Meng, T. and Matunaga, S.: Failure-Tolerant Control for Small Agile Satellites Using Single-Gimbal Control Moment Gyros and Magnetic Torquers, Acta Mechanica Sinica, 28 (2012), pp. 551-558.

6) Aristidou, A. and Lasenby, J.: FABRIK: A Fast, Iterative Solver for the Inverse Kinematics Problem, Graphical Models, 73 (2011), pp. 243-260.

7) Ford, K. and Hall, C.: Singular Direction Avoidance Steering for Control-Moment Gyros, J. Guidance, Control, and Dynamics, 23 (2000), pp. 648-656. 